\title{
Microstructure Controlled Shear Band Pattern Formation and Enhanced Plasticity of Bulk Metallic Glasses Containing in situ Formed Ductile Phase Dendrite Dispersions
}

\author{
C. C. Hays, C.P. Kim, and W. L. Johnson \\ Keck Laboratory of Engineering Materials, California Institute of Technology, Pasadena, California 91125
}

(Received 28 June 1999)

\begin{abstract}
Results are presented for a ductile metal reinforced bulk metallic glass matrix composite based on glass forming compositions in the $\mathrm{Zr}-\mathrm{Ti}-\mathrm{Cu}-\mathrm{Ni}-\mathrm{Be}$ system. Primary dendrite growth and solute partitioning in the molten state yields a microstructure consisting of a ductile crystalline Ti-Zr-Nb $\beta$ phase, with bcc structure, in a $\mathrm{Zr}-\mathrm{Ti}-\mathrm{Nb}-\mathrm{Cu}-\mathrm{Ni}-\mathrm{Be}$ bulk metallic glass matrix. Under unconstrained mechanical loading organized shear band patterns develop throughout the sample. This results in a dramatic increase in the plastic strain to failure, impact resistance, and toughness of the metallic glass.
\end{abstract}

PACS numbers: 81.40.-z, 81.05.Kf

$\mathrm{Zr}_{41.2} \mathrm{Ti}_{13.8} \mathrm{Cu}_{12.5} \mathrm{Ni}_{10} \mathrm{Be}_{22.5}$ (V1) exhibits an exceptional bulk metallic glass (BMG) forming ability that has motivated investigations of its mechanical behavior [1-3]. This alloy exhibits a $1.9 \mathrm{GPa}$ tensile yield strength, and a $2 \%$ elastic strain prior to failure under tensile or compressive loading. However, as in all metallic glasses, V1 specimens loaded in a state of uniaxial or plane stress fail catastrophically on one dominant shear band and show little global plasticity. Specimens loaded under constrained geometries (plane strain) fail in an elastic, perfectly plastic manner by the generation of multiple shear bands. Multiple shear bands are observed when the catastrophic instability is avoided via mechanical constraint, e.g., in uniaxial compression, bending, rolling, and under localized indentation. This behavior under deformation has limited the application of bulk metallic glasses as an engineering material.

This Letter presents results for a new class of ductile metal reinforced BMG matrix composites prepared via in situ processing. Under loading, the two-phase microstructure leads to spatial variations in elastic properties as well as the conditions for yielding, the ductile phase having a lower yield strain. The initiation and propagation of shear bands is controlled by the scale and geometry of the ductile phase dispersion with the result that deformation occurs through the development of highly organized patterns of regularly spaced shear bands distributed uniformly throughout the sample.

The compositions in the $\mathrm{Zr}$-Ti-Cu-Ni-Be system are compactly written in terms of a pseudoternary Zr-Ti- $X$ phase diagram, where $X$ represents the moiety $\mathrm{Be}_{9} \mathrm{Cu}_{5} \mathrm{Ni}_{4}$, characteristic of $\mathrm{Zr}_{41.2} \mathrm{Ti}_{13.8} \mathrm{Cu}_{12.5} \mathrm{Ni}_{10} \mathrm{Be}_{22.5}$. Results presented here are for alloys of the form $\left(\mathrm{Zr}_{100-x-z} \mathrm{Ti}_{x^{-}}\right.$ $\left.M_{z}\right)_{100-y} X_{y}$, where $M$ is an element that stabilizes the crystalline $\beta$ phase in Ti- or Zr-based alloys. The inset in Fig. 1 shows the $\mathrm{x}$-ray diffraction pattern for the nominal composition $\left(\mathrm{Zr}_{75} \mathrm{Ti}_{18.34} \mathrm{Nb}_{6.66}\right)_{75} X_{25}$; i.e., an alloy with $M=\mathrm{Nb}, z=6.66, x=18.34$, and $y=25$. The diffraction pattern was obtained with an INEL diffractometer (Co- $K_{\alpha}$ radiation) on the cross sectioned surface of a $25 \mathrm{~g}$ arc melted rod of roughly cylindrical diameter, $\phi=1 \mathrm{~cm}$.
The peaks shown [with (hkl) values labeled] are due to the bcc phase. A Nelson-Riley extrapolation yields a lattice parameter $a=3.496 \AA$ [4]. Upon cooling from the high temperature melt, the alloy undergoes partial crystallization by nucleation and subsequent dendritic growth of the $\beta$ phase in the remaining liquid. The remaining liquid subsequently freezes to the glassy state producing a twophase microstructure containing $\beta$-phase dendrites in a glass matrix. The final microstructure of a chemically etched specimen is shown in the scanning electron microscopy (SEM) image of Fig. 1. SEM electron microprobe analysis gives the average composition for the $\beta$-phase dendrites (light phase in Fig. 1) to be $\mathrm{Zr}_{71} \mathrm{Ti}_{16.3-}$ $\mathrm{Nb}_{10} \mathrm{Cu}_{1.8} \mathrm{Ni}_{0.9}$. Under the assumption that all of the $\mathrm{Be}$ in the alloy is partitioned into the matrix we estimate that the average composition of the amorphous matrix (dark phase) is $\mathrm{Zr}_{47} \mathrm{Ti}_{12.9} \mathrm{Nb}_{2.8} \mathrm{Cu}_{11} \mathrm{Ni}_{9.6} \mathrm{Be}_{16.7}$. Both are quoted

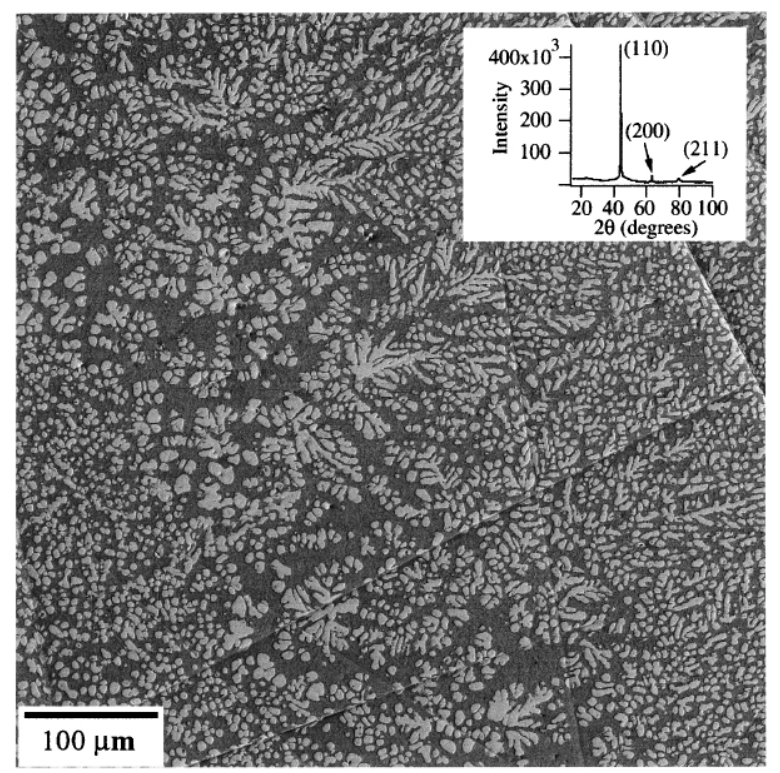

FIG. 1. SEM backscattered electron image of in situ composite microstructure $($ magnification $=200 \times)$. (Inset: X-ray diffraction pattern for $\left(\mathrm{Zr}_{75} \mathrm{Ti}_{18.34} \mathrm{Nb}_{6.66}\right)_{75} X_{25}$ in situ composite). 
in atomic percent. Microprobe analysis also shows that within experimental error (about \pm 1 at. \%), the compositions within the two phases do not vary. This implies solute redistribution and the establishment of chemical equilibrium within and between the phases following dendritic growth. DSC (differential scanning calorimetry) analysis of the heat of crystallization of the remaining amorphous matrix compared with that of the fully amorphous sample gives a direct estimate of the molar fractions (and volume fractions) of the two phases. This gives an estimated fraction of $\approx 25 \% \beta$ phase by volume $(\approx 75 \%$ amorphous phase). Direct estimates based on area analysis of the SEM image agree well with this estimate. The SEM image in Fig. 1 shows the fully developed dendritic structure of the $\beta$ phase. The dendritic structures are characterized by primary dendrite axes with lengths of 50-150 $\mu \mathrm{m}$ and radius of about $1.5-2 \mu \mathrm{m}$. Regular patterns of secondary dendrite arms with spacing $\lambda \approx 6-7 \mu \mathrm{m}$ are observed having radii somewhat smaller than the primary axis. The dendrite "trees" have a very uniform and regular structure.
The primary axes show some evidence of texturing over the sample as expected since dendritic growth tends to occur in the direction of the local temperature gradient during solidification. Details will be discussed in an upcoming publication. Transmission electron microscopy images of the dendrite/matrix interface show that the interface is atomically sharp and free of any third phases. It is an intimate amorphous/crystal interface.

A series of mechanical property tests were conducted on this alloy composition. These include quasistatic tensile and compression tests, Charpy impact, and three point bend tests on specimens prepared according to ASTM standards. The results of a noninstrumented three point bend test on a $2 \mathrm{~mm}$ by $1 \mathrm{~cm}$ by $3 \mathrm{~cm}$ beam shaped specimen show that the in situ composite undergoes a plastic strain $\varepsilon_{p} \approx 5 \%$ before failure. A monolithic metallic glass specimen of this size fails catastrophically by the propagation of a single shear band under identical loading. The mechanism responsible for this plasticity is seen in the SEM images of Fig. 2. Figure 2(a) shows a low
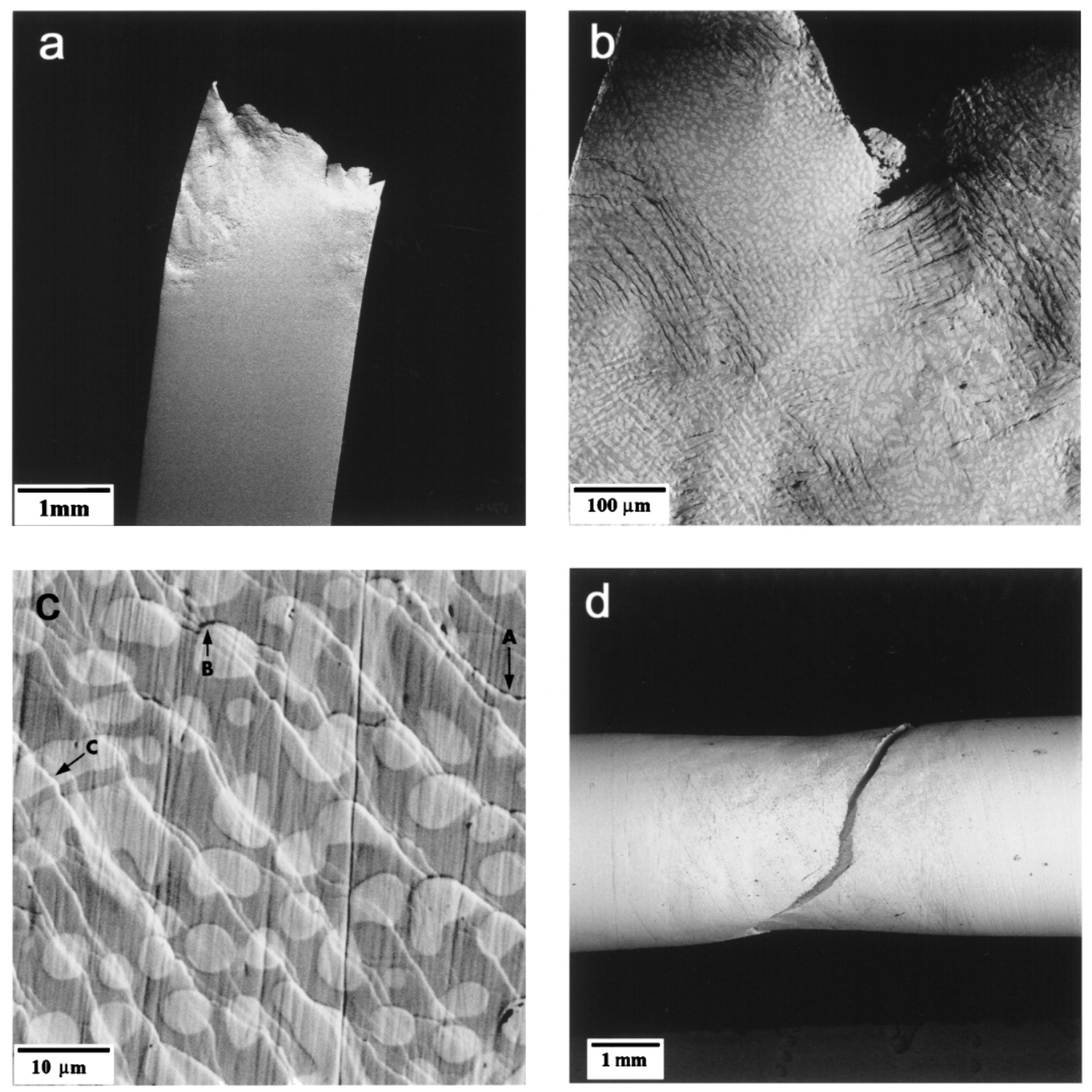

FIG. 2. Composite SEM images of mechanical test specimens: (a) bend test specimen (view normal to bend direction), (b) tensile failure region of bend test specimen, (c) shear band pattern array from compressive failure region of bend test specimen, and (d) deformed tensile specimen. 
magnification image of the bend specimen viewed normal to the bending direction. The permanent bend radius is seen along with a very rough fracture surface exhibiting mode I and mode II $\left(45^{\circ}\right.$ shear failure) failure surfaces. Figure 2(b) shows a higher magnification image of a region near the failure surface. Patterns of regularly spaced shear bands are seen throughout the region. Pattern domains have a spatial extent of $\approx 100 \mu \mathrm{m}$. Within each domain, a regular array of parallel shear bands is observed at a spacing of typically $\lambda \approx 7-8 \mu \mathrm{m}$. This spacing coincides with the secondary arm spacing of the $\beta$-phase dendrites. The bands occur on habit planes oriented roughly at $45^{\circ}$ to the tensile and compressive surfaces of bending (directions of maximum resolved shear stress in bending). Note that in areas where dendrite arms are normal to the surface, the shear bands preferentially propagate through the arms with slip apparently localized in a plane cutting through the axes of the arms. A variety of geometrical correlations can be seen between the shear bands and dendrites. A complete discussion is beyond the scope of this Letter. Figure 2(c) shows a still higher magnification image of a "domain" of shear bands where the dendrite arms are predominately normal to the surface. The shear bands propagate preferentially through many successive dendrite arms (often over the entire domain), occasionally initiate or terminate within the arms, and clearly propagate as localized bands through the $\beta$-phase arms. Coalescence voids are observed along individual shear bands (see point $A$ ) and at the interface between the ductile particles and the glassy matrix (see point $B$ ). Shear offsets at the ductile particle/glassy matrix interface of $\approx 0.5 \mu \mathrm{m}$ are observed with shear band widths $W$ of the same order (see point $C$ ). Therefore, the plastic strains within a single typical shear band are $\varepsilon_{p}^{\text {band }} \approx 1$. The total plastic strain can be estimated as $\varepsilon_{\text {total }} \approx W / \lambda_{s} \approx 4 \%$, in excellent agreement with the overall plastic strain in the specimen as determined by the plastic bend radius.

Figure 3 shows the compressive stress strain curve for a cylindrical in situ composite specimen with $3 \mathrm{~mm}$ diameter and $6 \mathrm{~mm}$ length. Under quasistatic loading (strain rate of $10^{-4}$ to $10^{-3} \mathrm{sec}^{-1}$ ) the material exhibits a Young's modulus $Y=110 \mathrm{GPa}$ and yields at $\sigma_{y} \approx 1.3 \mathrm{GPa}$, with a corresponding elastic strain limit $\varepsilon_{y} \approx 1.2 \%$. Beyond this limit the $\beta$ phase yields and deforms, and shear band patterns develop, as the glassy matrix is locally loaded beyond its critical shear stress. We believe that critical loading of the glass develops when plastic yield of the $\beta$ phase transfers local stress concentrations to the surrounding glass. On further deformation, an apparent strain hardening behavior is seen. We presume this to be related to the strain hardening behavior of the $\beta$ phase which tends to arrest local deformation. The apparent work hardening above a strain of 3\% follows a linear behavior described by the relation $\Delta \sigma_{y}=$ $2.84 \mathrm{GPa} /$ unit strain until an ultimate failure stress of $\sigma_{y}=1.7 \mathrm{GPa}$ is reached. Total strain (elastic + plastic) of over $8 \%$ is achieved prior to failure. In an identical test,

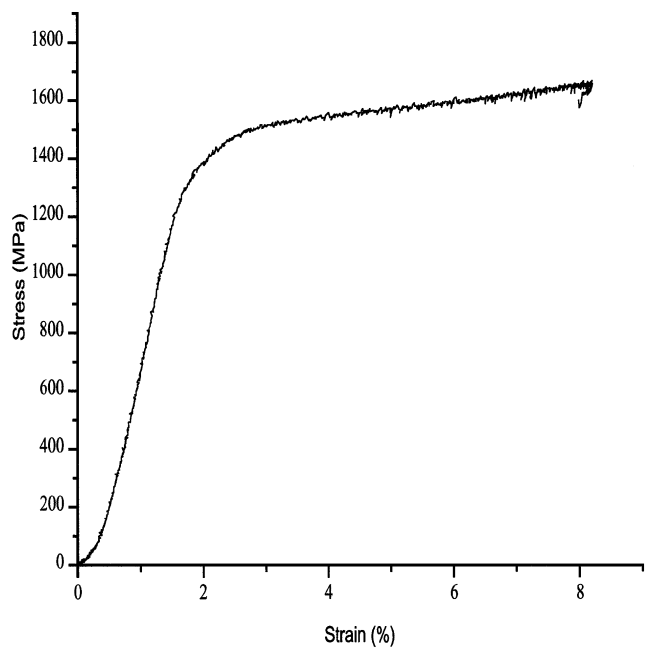

FIG. 3. Compressive stress strain curve for cylindrical in situ composite specimen.

a monolithic V1 metallic glass sample yields at a strain of $2 \%$, flows perfectly plastically (no measurable strain hardening), then fails catastrophically along a single or small number $(2-3)$ of shear bands with very little $(<1 \%)$ global plasticity. In the composite, failure occurred on a plane oriented at $\approx 45^{\circ}$ from the loading axis, a behavior similar to the failure mode of the monolithic BMG matrix. The composite fracture surface is, however, more complex showing some regions with "vein pattern" morphology characteristic of metallic glasses, and other large regions $(\approx 50-100 \mu \mathrm{m})$ which appear to have undergone extensive melting (large melt pools) and resolidification. This provides evidence of enormous heat dissipation in relatively large $(\approx 100 \mu \mathrm{m})$ local areas along the failure surface.

The bending and compression tests conducted impose geometric loading conditions that act to constrain shear bands and promote multiple shear banding. By contrast, loading under uniaxial tension is unstable and deformation unconfined. Metallic glass specimens loaded under tension exhibit essentially no ductility; failure occurs catastrophically by the propagation of a single shear band with plastic strain of $0.1 \%$ or less. Tensile tests were also conducted on the in situ composite. Standard ASTM tensile bars with $\phi=3 \mathrm{~mm}$ (diameter) and $L=20 \mathrm{~mm}$ (length) gauge sections were loaded at quasistatic strain rates of $10^{-4}$ to $10^{-3} \mathrm{sec}^{-1}$. A dramatically enhanced overall plastic strain of $\approx 5 \%$ (averaged over gauge length) was observed prior to failure. Clear necking of the tensile bar was observed, with a plastic strain of $15 \%$ in the necked region. The deformed tensile bar is shown in Fig. 2(d). The prepolished (prior to test) tensile specimen surface was examined in the SEM. Shear band pattern arrays were observed over the entire surface of the deformed tensile specimen. The shear band patterns were oriented on habit planes at $\pm 45^{\circ}$ from the tensile axis. The pattern periodicity as viewed normal to the slip direction was $\lambda_{s} \approx 6-7 \mu \mathrm{m}$, similar to those observed in the bend and compressive test specimens. Deformation under uniaxial tension apparently 
occurs in the same manner as under bending and compressive loading.

Charpy impact test specimens (a substandard $5 \times 5 \mathrm{~mm}$ cross section) prepared from the composite material have yielded an average Charpy impact toughness 250\% greater than that of monolithic BMG specimens prepared from compositions within the bulk glass forming range: $200 \mathrm{~kJ} / \mathrm{m}^{2}$ vs $80 \mathrm{~kJ} / \mathrm{m}^{2}$. The in situ composite fracture surfaces show a mixed fracture mode. Distinct shear lips, indicative of mode II or mode III fracture, are observed near the edges of the Charpy specimen. The central region of the Charpy specimen is relatively flat corresponding to plane-strain, mode I fracture. High magnification images of the fracture surface show the classic veinlike fracture morphology characteristic of the monolithic glass mixed with regions of ductile fracture. The ductile regions have a characteristic "dimple" type of morphology, indicating the coalescence of microvoids. The improved results for the Charpy test specimens indicate that deformation by shear band pattern formation is operant under the dynamic loading conditions of the Charpy test where strain rates are of the order $10^{3} \mathrm{sec}^{-1}$.

The initiation and propagation of localized shear deformation in metallic glasses has been the subject of both theoretical and experimental investigations for a number of years [5-7]. In metallic glasses, where models predict strain softening, and adiabatic heating produces dramatic thermal softening near the glass transition, one expects shear localization to be incipient and extreme, as is experimentally observed [8]. The idea of shear band confinement in metallic glass composites was first addressed in the work by Ling and Courtney, where thin metallic glass ribbons were sandwiched between thin brass plates [9].

In the new in situ composite of this Letter, the dendritic microstructure of the $\beta$ phase acts to seed the initiation of organized shear band patterns, confines the propagation of individual shear bands to domains having a spatial scale of the order of the primary dendrite axes length, and leads to shear band spacing which is related to the dendrite arm spacing $\lambda$. A natural variation of the local condition for slip is imposed by the dendritic microstructure which then results in formation of shear band arrays. From the observed shear bandwidth $W \approx 0.3-0.5 \mu \mathrm{m}$, the pattern spacing $\lambda \approx 6-7 \mu \mathrm{m}$, and the observation that the total slip across a band $S$, which is roughly equal to $W$ (total strain within a shear band is of order unity), we can estimate the global plastic strain arising from the shear band patterns to be $W / \lambda \approx 1 / 20=5 \%$ in excellent agreement with the "global" plastic strain observed in various mechanical tests above.

It is noteworthy that the matrix/dendrite interface in the present composites is atomically sharp, intimate, and apparently strong. This is evidenced by the fact that most interfaces remain intact during loading and yielding, al- though separation of the matrix/dendrite interface is also occasionally observed. This allows effective transfer of stress from the $\beta$ phase to the amorphous matrix when yielding occurs initially in the $\beta$ phase and may play a key role in seeding the formation of multiple shear bands. In crystalline composite materials it is well known that shear localization can have microstructural or thermal origins [10]. The thermal and/or mechanical coupling between phases provides a positive driving force for shear localization and thus promotes the formation of shear bands at specific microstructural sites. The ductile phase in the new in situ composite may have a dual role in the response to mechanical loading; they may serve as heterogeneous sites for the initiation of individual shear bands, while at the same time act as attraction or pinning centers during shear band propagation.

The present results for the new in situ ductile phase/ BMG matrix composite are unique and demonstrate a new physical phenomena which arises when the microstructural length scale (dendrite tip radius, arm spacing, etc.) and critical mechanical length scales (shear band width and spacing, etc.) are suitably chosen. The shear band pattern formation into regular arrays as controlled by the microstructure illustrates the cooperative interplay of the microstructure with the mechanism of mechanical deformation. This opens the possibility of producing an entirely new class of high strength, tough, impact and fatigue resistant materials which combine the high strength of metallic glass with the ability to undergo plastic deformation under unconfined or otherwise unstable loading conditions.

The authors acknowledge the U.S. Department of Energy (Grant No. DEFG-03-86ER45242) for the support of C.C. H. We acknowledge the Army Research Office (Grant No. DAAG55-98-1-0305) for the support of C. P. K. We also thank Wayne Waller and Carolyn Patterson of the Caltech Digital Media Center for their assistance.

[1] H. A. Bruck, T. Christman, A. J. Rosakis, and W. L. Johnson, Scr. Metall. 30, 429 (1994).

[2] H. A. Bruck, A. J. Rosakis, and W. L. Johnson, J. Mater. Res. 11, 503 (1996).

[3] C. J. Gilbert, R. O. Ritchie, and W. L. Johnson, Appl. Phys. Lett. 71, 476 (1997).

[4] L. Azaroff and M. J. Buerger, The Powder Method in X-Ray Crystallography (McGraw-Hill, New York, 1958), p. 238.

[5] F. Spaepen and D. Turnbull, Scripta Metall. 8, 563 (1974).

[6] J. J. Gilman, J. Appl. Phys. 46, 1625 (1975).

[7] J.C.M. Li, in Metallic Glasses, Proceedings of the Materials Science Division of the American Society for Metals (American Society for Metals, Metals Park, OH, 1976).

[8] F. Spaepen and D. Turnbull, Scr. Metall. 8, 563 (1974).

[9] Y. Ling and T.H. Courtney, Metall. Trans A 21, 2159 (1990).

[10] M. A. Meyers, J. Phys. (Paris), Colloq. 4, C8-597 (1994). 